\title{
Uso actual de las tecnologías de información y comunicación en la educación médica
}

\author{
Current use of information and communication technologies in medical education
}

\author{
Manuel Pérez Martinot 1, ; $;$
}

\section{RESUMEN}

Las tecnologías de información y comunicación siguen en constante desarrollo y su aplicación se ha generalizado. La educación medica no es ajena a este fenómeno por lo que es importante que tanto profesores como alumnos adopten estas herramientas para conseguir un proceso enseñanza-aprendizaje de calidad. Con este objetivo, se realizó una revisión bibliográfica actualizada de los conceptos más relevantes con el tema y de uso actual en la educación médica. Se puede apreciar una tendencia a la incorporación de las nuevas tecnologías en la educación médica rompiendo con los esquemas tradicionales de transmisión de conocimientos. Los estudiantes deberán utilizar la computadora como un medio de investigación, para obtener, generar e intercambiar información científica más actualizada y continuar su proceso de educación médica.

PALABRAS CLAVE: Informática, software, enseñanza, multimedia. (Fuente: DeCS BIREME).

\section{SUMMARY}

The information and communication technologies are still in constant development and widespread application. Medical education is no stranger to this phenomenon so it is important that both teachers and students adopt these tools for a quality teaching-learning process. To this end, an updated list of the most relevant concepts with the subject and current use in medical education literature review was conducted. We can see a trend towards the incorporation of new technologies in medical education breaking with traditional patterns of transmission of knowledge. Students will use the computer as a means of investigation to obtain, generate and exchange latest scientific information and continuing medical education process.

KEY WORDS: Informatics, software, teaching, multimedia. (Source: MeSH NLM).

\section{INTRODUCCIÓN}

El proceso de enseñanza-aprendizaje de la Medicina se ha enriquecido con el uso de las computadoras y de las tecnologías de la información y comunicación (TIC) que favorecen la aplicación de materiales educativos computarizados (MEC) especialmente diseñados para estos fines.

Los centros dedicados a la enseñanza de la medicina están continuamente buscando, desarrollando y aplicando nuevos MEC que los mantenga a la vanguardia como formadores de médicos de gran categoría y así poder recibir el reconocimiento correspondiente a nivel nacional e internacional. Por esta razón, es importante que, tanto docentes como estudiantes, conozcan las diferentes herramientas tecnológicas y su disponibilidad en el medio para apoyarse en ellas y facilitar su desempeño en las aulas de estudio.

El objetivo de este trabajo fue revisar los conceptos básicos de las TIC y su uso actual en la educación médica. Para este fin, se hizo la búsqueda bibliográfica 
para la identificación de estudios, siguiendo la siguiente metodología:

- Búsqueda electrónica de estudios publicados: Se realizó la búsqueda en MEDLINE/Pubmed, Cochrane Central Register of Controlled TrialsCENTRAL in The Cochrane Library y Global Health Library (incluye LILACS); se utilizaron las palabras clave informática, software, enseñanza, multimedia, informatic, teaching. Esta búsqueda se limitó a utilizar sólo estas estrategias para poder estandarizar los métodos en los distintos motores de búsqueda y asegurar la relación entre artículos. La búsqueda inicial incluyó todo tipo de artículos y no hubo restricción en cuanto al año y lenguaje de publicación. En una segunda revisión, se priorizó todas aquellas referencias de los últimos años.

- Búsqueda electrónica de estudios no publicados: Para buscar la literatura gris de estudios no publicados se buscó en las bases de datos Web of Science y Google Scholar utilizando una estrategia de búsqueda similar a la mencionada anteriormente.

- Otras fuentes: De los estudios recolectados, se realizó una búsqueda de las referencias dentro de los estudios relevantes para identificar literatura de importancia adicional. Otra de las maneras en las que se obtuvo información fue mediante la búsqueda manual dentro de revistas cuyos temas publicados eran relevantes para el tema de esta revisión.

- Combinando resultados de la búsqueda: Toda la literatura encontrada fue almacenada en una sola base de datos bibliográfica. Los estudios duplicados, fueron eliminados.

\section{LAS TECNOLOGIAS DE LA INFORMACION Y COMUNICACION (TIC)}

En los últimos años hemos sido testigos de la evolución de las TIC y de su integración en diferentes aspectos de la educación. Conocidas desde hace años como: “...un conjunto de aparatos, redes y servicios que se integran o se integrarán a la larga, en un sistema de información interconectado y complementario..." (1), conforman un sistema basado en las telecomunicaciones, la informática y la tecnología audiovisual.

Como parte importante de la informática, caracterizada por su gran desarrollo tanto en hardware como en software, disponemos del multimedia, el cual nos permite producir, transmitir, editar y almacenar la información integrada de diferentes formatos de manera efectiva y utilizar este producto como material educativo. En este grupo destacan también, el internet, los bancos de información interactiva, los servicios de mensajería electrónica, y otros que son empleados así mismo, en el proceso educativo.

\section{Materiales educativos computarizados (MEC)}

Es todo aquel aplicativo que "apoya directamente al proceso de enseñanza-aprendizaje" (2) y a la investigación. Algunos autores sostienen que este concepto debe ser más dinámico y no debe quedarse en su concepción inicial, sino que "debe evolucionar según las necesidades, expectativas y realidades del contexto actual, sin las limitaciones de espacio ni tiempo que representa el trabajo presencial en grupo (3).

Las clases tradicionales, en la que el profesor dictaba su clase magistral y el alumno era simplemente un receptor, debe ir cambiando. Con estos materiales, se debe procurar crear entornos de aprendizaje participativos donde tanto el alumno como el profesor tengan un rol activo.

El alumno debe ser parte activa en la construcción del conocimiento. Se debe fomentar su creatividad,

Tabla 1. Ventajas y desventajas de los materiales educativos computarizados (MEC).

\begin{tabular}{|c|c|}
\hline VENTAJAS & DESVENTAJAS \\
\hline - Permiten el logro de los objetivos de aprendizaje. & $\begin{array}{l}\text { - El diálogo alumno-profesor tradicional es reemplazado por } \\
\text { el lenguaje virtual }\end{array}$ \\
\hline - Facilitan la producción de la enseñanza & $\begin{array}{l}\text { - En paquetes cerrados, no hay lugar para el desarrollo de la } \\
\text { creatividad. }\end{array}$ \\
\hline $\begin{array}{l}\text { - Cumple con las exigencias y expectativas de los } \\
\text { programas de estudio vigentes. }\end{array}$ & - Los medios virtuales desplazan al libro tradicional \\
\hline - Usan herramientas virtuales actuales. & $\begin{array}{l}\text { - Están expuestos a virus informáticos en la red. Es posible } \\
\text { perder o dañar la información }\end{array}$ \\
\hline - Promueve la participación del alumno y la investigación. & - Las fuentes de información no siempre son confiables \\
\hline - Ayudan en la construcción del conocimiento. & \\
\hline
\end{tabular}


juicio crítico para la resolución de casos problema y la posibilidad de trabajar en grupo. Por el otro lado, el profesor debe actuar como presentador, administrador y facilitador del aprendizaje. Así mismo, los MEC al ser herramientas pedagógicas tienen principios metodológicos factibles de ser evaluados durante el desarrollo de las actividades. Las ventajas y desventajas de los MEC, se resumen en la tabla 1.

\section{a) Criterios para el uso de MEC}

Hay algunos criterios que se deben tener en cuenta cuando se construye un programa, cualquiera sea el área del conocimiento, empleando materiales educativos. Estos son (4):

- Cultura: considerar que la cultura es amplia y compleja, con múltiples interacciones entre los sujetos y se transmite de una generación a otra.

- Selección: se debe saber bien lo que se desea enseñar para de esta manera elegir adecuadamente los contenidos del programa.

- Estructura: la confección del currículo guarda relación estrecha con el contenido del programa.

- Metodología: considerar la más adecuada para transmitir los conceptos al alumno.

- Evaluación: de la calidad y eficiencia de los productos educativos.

\section{b) Software Educativo (SE)}

En términos generales, los $\mathrm{SE}$ son programas informáticos o aplicaciones que facilitan el proceso enseñanza-aprendizaje. Son herramientas pedagógicas que ayudan a la adquisición de conocimientos y al desarrollo de habilidades. Sus características principales son: "estar orientados a la docencia, utilizar una computadora como medio de soporte, ser de uso amigable e interactivo con el usuario" (5).

La estructura de un SE está conformada básicamente por tres módulos: "un sistema de input/ output que gestiona la comunicación con el usuario, la base de datos que contiene debidamente organizados los contenidos informativos del programa y el motor, que gestiona las actuaciones del ordenador y sus respuestas a las acciones de los usuarios" (6).

Una vez identificado el problema educativo a resolver y asegurar que la computadora ofrece ventajas cualitativas sobre otros recursos para solucionarlo, se inicia recién el proceso de creación de un SE. Luego, se debe realizar una búsqueda y análisis de la bibliografía de la materia a estudiar, de los procesos de enseñanza y de lo relacionado con los aspectos técnicos de programación.

Las clasificaciones de los SE son diversas. Una de ellas clasifica los programas en Directivos o No Directivos, considerando el grado de aciertos o desaciertos de los alumnos. Así, en los programas directivos, se hacen preguntas y el programa determina cuándo el alumno comete un error, mientras que en los programas no directivos la computadora procesa los datos introducidos por el alumno y muestra las consecuencias de su respuesta, sin juzgar las acciones del mismo. Otra clasificación los divide en programas Abiertos y Cerrados, de acuerdo a la posibilidad de modificar y adecuar sus contenidos según la materia de estudio. Sin embargo, la que tiene en cuenta "el grado de control del programa sobre la actividad de los alumnos y la estructura de su algoritmo", es la que resulta más útil y clara a los profesores $(7,8)$, esta clasifica a los $\mathrm{SE}$ en: tutoriales, tutores inteligentes, simuladores, micromundos e hipertextos e hipermedias:

- Tutoriales. Se usan diálogos a través de los cuales el tutor hace preguntas al alumno y la actividad de éste es controlada por el computador. Tienen la ventaja de sustituir al profesor en muchas tareas y ofrecer más oportunidades a los alumnos menos preparados. Su principal desventaja es la de no resultar muy atractivos a los alumnos más avanzados (8).

- Tutores inteligentes. Se trata de sistemas expertos que imitan la capacidad mental del hombre y hacen juicios acerca de un problema más rápidamente que el ser humano. En la medicina tiene gran utilidad en los casos de diagnóstico.

- Simulaciones. Aquí el control del proceso lo tiene el alumno y no el computador. El estudiante debe ser capaz de descubrir las reglas en la simulación de determinado entorno mediante la experimentación. El computador es capaz de crear un micro mundo con el cual el alumno tiene la posibilidad de interactuar, simulando procesos físicos o biológicos que requerirían en la vida real de experimentos peligrosos o muy costosos, o que se producirían en tiempos muy breves o excesivamente largos (9).

- Hipertextos e hipermedia. Organizan la información de manera no lineal, dándole la posibilidad al estudiante de poder consultar la información según sus necesidades y experiencia previa en el tema (9).

En la práctica, lo que encontramos son programas que resultan de la mezcla de los diferentes tipos de 
SE. Por lo tanto, no son excluyentes entre sí, sino por el contrario, pueden integrar características de varios de ellos para responder a un problema educativo determinado.

Es posible establecer una relación entre los diferentes tipos de SE y los modos de aprendizaje. "Los tutoriales están relacionados con modelos conductistas; los tutores inteligentes, con modelos cognitivos y los simuladores, así como los hipertextos e hipermedias, con modelos constructivistas" (7).

No hay duda que "el uso de SE es útil por lo que aporta al proceso de enseñanza aprendizaje y porque prepara al futuro médico en el trabajo con las nuevas tecnologías que van a serle esenciales para estar actualizado y ser eficiente en su actividad profesional" (7). Sin embargo, para su mejor aprovechamiento en el medio docente, es necesario disponer de SE de calidad, medidos en términos del conocimiento que sean capaces de transmitir, debe ser utilizado fácilmente, los códigos y símbolos presentados en la pantalla deben ser comprensibles, debe tener una respuesta rápida a las acciones del usuario y, el navegador utilizado debe ser amigable, sencillo y comprensible (7).

\section{c) Multimedia}

Consiste en el uso de diversos medios (voz, texto, animación, videos, gráficos, datos, etc.) para presentar y transmitir información que se puede reproducir en un computador con el hardware adecuado (por ejemplo, tarjetas de sonido y video, monitores). Su principal característica es la interacción, la cual permite al usuario una mayor sensación de realismo debido a la incorporación de audio, video, imágenes fijas o animadas, incluso en tercera dimensión que puede adaptarse a cualquier SE especialmente a aquellos relacionados a educación médica. El usuario, aún con escasos conocimientos de informática, tiene la capacidad de navegar, interactuar, crear y comunicarse de una manera más fácil, didáctica y atractiva. Estas aplicaciones multimedia pueden estar almacenadas digitalmente en discos ópticos o discos compactos o CD-ROM, por sus siglas en inglés Compact Disc Read-Only Memory (para su uso off-line) o estar almacenados en páginas de Web (uso on-line) (10).

No existe un sistema multimedia mejor que otro; su elección dependerá de la finalidad que tiene la aplicación multimedia (10). Los sistemas de navegación multimedia más frecuentes en relación a la intervención del usuario, son los siguientes:
- Lineal: Para acceder a los diferentes módulos de la aplicación, el usuario únicamente puede seguir un determinado camino ("sistema de navegación lineal o secuencial"). Esta estructura es utilizada en gran parte de las aplicaciones multimedia de ejercitación y práctica o en libros multimedia. (10)

- Reticular: El usuario es libre para seguir diferentes caminos cuando navega por el programa utilizando la capacidad de hipertexto. Es la indicada para las aplicaciones orientadas a la consulta de información, por ejemplo, para la realización de una enciclopedia o un atlas electrónico (10).

- Jerarquizado: Este sistema es muy utilizado pues combina las ventajas de los dos sistemas anteriores.

\section{d) E-learning}

Desde sus inicios en la década de los ochenta, el e-learning o aprendizaje electrónico, ha ido evolucionado vertiginosamente. Con la aparición de la computadora personal, se comenzó a utilizar esta tecnología con fines didácticos como material de apoyo en las clases (enseñanza asistida por computadora). La mejora de la capacidad de las computadoras y el surgimiento de programas interactivos con la posibilidad de integrar audiovisuales, permitió elaborar materiales con contenidos multimedia (multimedia educativa), que tuvo su vigencia hasta mediados de los noventa. En la segunda mitad de los años noventa, se consolida el Internet y se "inicia la era de la educación a distancia apoyada en páginas Web educativas. Esto favoreció una mayor autonomía del estudiante a través del estudio independiente, y el proceso de enseñanza se centró en el aprendizaje colaborativo. A principios del presente siglo se incorpora la gestión de la organización educativa y las técnicas de gestión del conocimiento a través del uso de las TIC" (11), creando estándares de calidad educativa que facilitan el intercambio de información y contenidos entre plataformas de diferentes instituciones. En la tabla 2, se resume la evolución del e-learning.

Muchos son los beneficios del e-learning, como su flexibilidad en el tiempo, mayor oferta para acceder a la educación, contenido del material educativo de mejor calidad, posibilidad de uso de simuladores, entre otros (11). Sin embargo, los costos de su implementación, la ausencia de equipos suficientes para favorecer su accesibilidad y el bajo nivel de capacitación digital de un grupo considerable de profesores, son algunas de las limitaciones que presenta. Además, se pierde el contacto directo entre el alumno y el profesor y, la falta de dedicación de este último, al diseño de contenidos y seguimiento de las acciones educativas (11). 
Tabla 2. Evolución del e-learning en el tiempo.

\begin{tabular}{|c|c|c|c|c|}
\hline 1984 & 1989 & 1994 & 1999 & 2004 \\
\hline Centrado en el docente & Era multimedia & E-learning Primera Ola & $\begin{array}{l}\text { Segunda Ola Más } \\
\text { tecnología de alto } \\
\text { desempeño }\end{array}$ & $\begin{array}{c}\text { Tercera Ola } \\
\text { Colaboración y } \\
\text { construcción social }\end{array}$ \\
\hline $\begin{array}{l}\text { Enseñanza asistida por } \\
\text { computador }\end{array}$ & $\begin{array}{l}\text { Material multimedia } \\
\text { transportable, } \\
\text { interactivo, } \\
\text { amigable }\end{array}$ & $\begin{array}{l}\text { Internet, correo } \\
\text { electrónico, buscadores, } \\
\text { lenguaje HTML, } \\
\text { reproductores, } \\
\text { integración de audio, } \\
\text { video, JAVA }\end{array}$ & $\begin{array}{l}\text { Enseñanza asistida por } \\
\text { computador basado en } \\
\text { la Web }\end{array}$ & $\begin{array}{l}\text { Páginas personales } \\
\text { (Blogs), flogs, } \\
\text { Enciclopedias (wikis), } \\
\text { Youtube, Blogger, } \\
\text { Wordpress }\end{array}$ \\
\hline $\begin{array}{l}\text { Materiales lineales no } \\
\text { interactivos }\end{array}$ & & Software propietario & Tutoría en tiempo real & $\begin{array}{l}\text { Hacia la web como } \\
\text { sistema operativo }\end{array}$ \\
\hline \multirow[t]{6}{*}{$\begin{array}{l}\text { Control de los } \\
\text { contenidos }\end{array}$} & & $\begin{array}{l}\text { Tutorías por correo } \\
\text { electrónico }\end{array}$ & $\begin{array}{l}\text { Servicio en línea al } \\
\text { alumno }\end{array}$ & $\begin{array}{l}\text { Mundos virtuales de } \\
\text { aprendizaje: avatares }\end{array}$ \\
\hline & & $\begin{array}{l}\text { Cursos multimedia por } \\
\text { internet }\end{array}$ & Contenido para la Web & $\begin{array}{l}\text { Entornos de aprendizaje } \\
\text { multidimensional y } \\
\text { colaborativo }\end{array}$ \\
\hline & & Video y teleconferencia & Open Source* & Aprendizaje abierto \\
\hline & & Logro de tareas & $\begin{array}{l}\text { Entornos de aprendizaje } \\
\text { multidimensional }\end{array}$ & Personalización \\
\hline & & & Adaptación pedagógica & \\
\hline & & & Grupos personalizados & \\
\hline
\end{tabular}

*Código abierto o software libre (Fuente: Adaptado de Gatica y Rosales (11)

“Tim O’Reilly y Dale Dougherty crearon en el 2004 el término Web 2.0 refiriéndose a una plataforma de trabajo abierta y red social que nace del internet y cuya característica sería la participación y colaboración. Este concepto reemplaza al de web tradicional donde el usuario tenía un rol más pasivo, simplemente de consumidor de información. Actualmente, el concepto de Web 2.0 ha evolucionado hacia el de Web en la nube, donde el usuario tiene acceso a material y recursos diversos independientemente del lugar donde se encuentre vía internet (informática ubícua)" (11).

Los estudiantes de medicina ya poseen alguna experiencia en al menos algunas herramientas web 2.0, $\mathrm{y}$ tienen una mayor ventaja respecto a las habilidades tecnológicas de sus profesores. "Estos jóvenes son considerados la generación Net, porque nacieron a partir de los años ochenta y tienen un alto nivel de interacción y de uso de las TIC en su vida cotidiana.
Esta generación de estudiantes demanda cambios en la forma de aprender y de enseñar la medicina..." (12). Es preciso, entonces, "modificar los procesos de instrucción, los recursos para mediarla y los métodos de aprendizaje para que se ajuste a sus necesidades educativas" (13). Hay que considerar que hoy en día, los estudiantes de medicina "son nativos digitales mientras que los profesores que nacieron antes de esa generación son inmigrantes digitales, y se están formando en el uso de las tecnologías y adaptándose a una nueva cultura para acceder, distribuir y compartir el conocimiento" (14).

Hay aplicaciones que potencian la formación médica a distancia. Entre estas "están los blogs, las wikis, los podcast, videoblogs y las redes sociales" (15) y han sido las preferidas para la implementación del e-learning en procesos educativos, generalizándose por la facilidad de uso, su portabilidad y la conectividad 
que ofrecen entre ellas (16). En la tabla 3, se detallan algunas de las diferentes herramientas disponibles actualmente utilizadas en e-learning en la educación médica.

El uso de e-learning no debe situar a la tecnología por encima de la pedagogía y la didáctica. No todos los procesos educativos pueden desarrollarse bajo tecnologías virtuales. Debemos tener en cuenta, entre otras cosas, la infraestructura tecnológica disponible en el centro de estudios y la capacitación en el uso de TIC modernas de los docentes para adoptar procesos de enseñanza bajo esta plataforma. (17)

Hoy en día, el e-learning constituye una opción estratégica básica para las instituciones universitarias de enseñanza de ciencias médicas que necesitan información actualizada para sus profesores y alumnos. La propuesta debe ir dirigida a crear bancos de materiales educativos disponibles en la web, de acceso universal, donde se garantice la calidad de los mismos, donde los profesores y alumnos con menos recursos puedan disponer de los recursos para el desarrollo de las diferentes asignaturas del programa curricular. De esta manera se superaría el obstáculo económico para la utilización universal del e-learning. Obviamente se tendría que organizar toda una política para uso, respetando los derechos legales de autor correspondientes y compatibilidades entre las diferentes plataformas utilizadas.

\section{Las TIC en la educación médica}

El reto actual de las universidades y facultades de ciencias de la salud radica en preparar a sus estudiantes y docentes a adaptarse a los cambios de manera rápida, efectiva y al menor costo. Nuevas tareas y responsabilidades les esperan: al alumno "aprender a usar las nuevas tecnologías y usarlas para aprender"; al docente, utilizar las TIC como medio para enseñar y promover en los alumnos la adquisición de nuevos conocimientos como parte de su formación como futuros médicos (18).

Es común encontrar computadoras en las aulas de educación médica, sin embargo, su utilización es todavía muy limitada y son pocos los docentes que la emplean de rutina y menos aún con fines educativos.

El empleo de las computadoras en el proceso docente tiene cuatro objetivos: " lograr el dominio del aprendizaje por reforzamiento y ejercitación; realizar procesos de aprendizaje por descubrimiento; generar procesos de búsqueda en contexto de interacción; $y$, favorecer el proceso de construcción del conocimiento" (19).

En los últimos años el mercado educativo ha sido invadido por gran cantidad de materiales educativos computarizados, sin embargo, aún no se consigue una alta oferta de software educativo de buena calidad. Los docentes deben ser los encargados de seleccionar y evaluar estos recursos para así poder integrarlos en su estrategia educativa (20).

Hay consenso entre los autores de la importancia del empleo de la computadora en la educación médica con el fin de capacitar al estudiante para su futura vida profesional y crear una cultura informática respecto al uso de las nuevas tecnologías. El uso de la computadora en la educación médica se aplicaría en cuatro niveles (21):

1. En la adquisición de conocimientos básicos, por ejemplo, con programas que capacitan al estudiante en procesos fisiopatológicos.

Tabla 3. Recursos de e-learning en Medicina.

\begin{tabular}{ll}
\hline & \multicolumn{1}{c}{ Herramientas } \\
\hline Organizadores de Información & LiveBinders, Mendeley \\
Buscador de imágenes y textos médicos & Yale image finder, Sciencerroll search, Gdocu, Pubget, Pubgle, JURN, JANE \\
Sindicación de contenidos (RSS) & $\begin{array}{l}\text { Clinical reader, bloglines, NetVibes, Webbicina-PeRSSonalized, MedWorm, } \\
\text { Google Reader, Jumbra }\end{array}$ \\
Redes sociales & Asklepios, PatientsLikeMe, Mydiabetes, ning.com, vi.vu, mySpace \\
Publicaciones en línea & PlosOne, Citeulike \\
Mezcladores de aplicaciones (Mashup) & HealthMap, EpiSPIDER, Avian flu, Vimo, Berkeley-Area Doctors, PubMed, \\
& Faceoff, Biowizard, eTBLAST, HubMed, OODesk, Glide \\
Wikis médicas & Radiopaedia.org, HealthGrid, AskDrWiki, Wiki Health, Medical Education, \\
Software para recursos de aprendizaje & Harvard medical school wiki, Med Revise \\
\hline
\end{tabular}

Fuente: Gatica y Rosales (11) 
2. En el adiestramiento clínico: mediante el uso de simuladores que permiten al estudiante aplicar sus conocimientos en situaciones análogas a la vida real.

3. En el desarrollo de prácticas de laboratorio: simulando prácticas y experimentos repetidos, económicos y muy seguros para el estudiante.

4. En el desarrollo de modelos educativos, que permitan la estructuración del conocimiento mediante sistemas expertos. Por ejemplo, puede desarrollarse un sistema computacional de fisiopatología en base a conocimientos y estrategias de aprendizaje flexibles para el alumno.

Actualmente, muchos centros de enseñanza de la medicina han implementado asignaturas que utilizan la modalidad de enseñanza mixta (tradicional y virtual) $y$, en algunos otros se imparten contenidos exclusivamente en línea a través de módulos interactivos de aprendizaje en plataformas cerradas (Blackboard, e-educativa) o abiertas (Moodle) (22), que permiten el aprendizaje en pequeños grupos, aprendizaje basado en problemas y presentación de casos clínicos. Hay reportes en la literatura en los cuales se afirma que el e-learning mejora el desempeño académico de los estudiantes a diferencia de quienes se forman con métodos de enseñanza tradicional (23).

\section{CONCLUSIONES}

Se puede apreciar una tendencia a la utilización de las TIC en la educación médica. Su constante avance crea la necesidad de mantenernos bien preparados y actualizados. Como profesores tenemos la responsabilidad y la obligación de incorporarlas en nuestras estrategias docentes para una educación de mayor calidad y romper con los esquemas tradicionales de transmisión de conocimientos.

Los alumnos egresados de nuestras facultades tendrán que ser capaces de manejar el amplio volumen de información que se genera cada vez más y se difunde de inmediato, quedándose tan sólo con lo verdaderamente significativo. El reto que se nos presenta como docentes es encontrar la manera de utilizar adecuadamente estos recursos que nos están trayendo las nuevas tecnologías de información y comunicación y, a los alumnos, de saber aprovecharlas para ser gestores de su propio conocimiento.

Finalmente, el conocimiento de computación e informática en la educación médica no debe limitarse a una asignatura dentro del currículo. Los estudiantes se deberán preparar para ser los médicos del mañana, quiénes tendrán que utilizar la computadora como un medio de investigación, para obtener, generar e intercambiar información científica más actualizada y para continuar su proceso de educación médica continua.

\section{Correspondencia:}

Manuel Pérez Martinot

manuel.perez.m@upch.pe

\section{REFERENCIAS BIBLIOGRÁFICAS}

1. Gómez C. Nuevas tecnologías de comunicación. Ciudad de México: Editorial Trillas; 1991.

2. Galvis-PanquevaAH. Software educativo multimedia. aspectos críticos no seu ciclo de vida. Revista Brasileira de Informática na Educação. 1997; 1: 9-18. DOI: http://dx.doi.org/10.5753/rbie.1997.1.1.9-18

3. Organización de las Naciones Unidas. Comisión Económica para América Latina y el Caribe, Organización de Estados Iberoamericanos para la Educación la C y la C, Secretaria General Iberoamericana. Metas educativas 2021: la educación que queremos para la generación de los bicentenarios. Documento final: síntesis. OEI; 2010.

4. Moya AM. Las nuevas tecnologías en la educación. Innovación y experiencias educativas. 2009; 24: 1-9.

5. Castro R. El software educativo en el entorno de los medios de enseñanza. Ciencias Holguín. 2008; 14(3):1-6.

6. Anaya S, Hernández U. Los materiales educativos computarizados (MEC) en la era de las Redes Sociales. Lima: EDUSOL, Quinto Encuentro en Línea de Educación Cultura y Software Libres; 2009.

7. Marqués, P. (1995). Software educativo: Guía de uso y metodología de diseño. Barcelona: Estel.

8. Vidal M, Gómez F, Ruiz A. Software educativos Rev Cuba Educ Medica Super. 2010; 24(1): 97-110. (Citado el 25 de junio de 2016). Disponible en: http:// scielo.sld.cu/pdf/ems/v24n1/ems12110.pdf

9. Ruiz-Parra AI, Angel-Müller E, Guevara O. Clinical simulation and virtual learning. Complementary technologies for medical education. Rev Fac Med. 2009; 57(1): 67-79.

10. Ortíz CB. Desarrollo de aplicaciones multimedia interactivas. Valencia: Unidad de Tecnología Educativa, Universidad de Valencia;. 2006. p. 1-10.

11. Gatica F, Rosales V. E-learning en la educación médica. Rev Fac Med. 2012;55:27-37.

12. Niemi PM. Medical students' professional identity: self-reflection during the preclinical years. Med Educ. 1997;31(6):408-15. doi: http://doi.wiley. com/10.1046/j.1365-2923.1997.00697.x 
13. Oblinger DG, Oblinger JL. Educating the Net Generation. Washington DC: EDUCAUSE; 2005.

14. Prensky M. Digital Natives, Digital Immigrants. On the Horizon. 2001; 9(5):1-3. (Citado el 25 de junio de 2016) Disponible en: http://www.marcprensky. com/writing/Prensky\%20-\%20Digita1\%20 Natives, \%20Digital\%20Immigrants\%20-\%20Part1. pdf

15. Ellaway R. E-learning: is the revolution over? Med Teach. 2011;33(4):297-302. doi: 10.3109/0142159X.2011.550968.

16. Khogali SEO, Davies DA, Donnan PT, Gray A, Harden RM, Mcdonald J, etal. Integration of e-learning resources into a medical school curriculum. Med Teach. 2011;33(4):311-8. doi: 10.3109/0142159X.2011. 540270

17. Harden RM. Myths and e-learning. Med Teach. 2002; 24(5):469-72. Doi: https://doi.org/10.3109/01 42159X.2011.540270

18. Sáez JM. Utilización de las TIC en el proceso de enseñanza aprendizaje, valorando la incidencia real de las tecnologías en la práctica docente. Docencia e Investigación. 2010; 35:183-204.
19. Aguilar J. ¿Capacitar en computación o cómputo para educar? RED. 1995;(53):23-5.

20. UNESCO. Estándares UNESCO de competencia en tic para docentes. Washington DCX: ONU para la Educación, la Ciencia y la Cultura; 2008.p.1-28.

21. Gutiérrez R, Hernández A. La educación médica y la computadora. Ciudad de Mexico: Informática en medicina; 1991.

22. Punie Y, Ala-mutka K. Future learning spaces: new ways of learning and new digital skills to learn. Digital Kompetance. 2007; 2: 210-25.

23. Means B, Toyama Y, Murphy R, Bakia M, Jones $\mathrm{K}$. Evaluation of evidence-based practices in online learning: Ameta-analysis and review of online learning studies. Washington DC: U.S. Department of Education; 2009. (Citado el 25 de junio de 2016). Disponible en: http://files.eric.ed.gov/fulltext/ ED505824.pdf

Recibido: 22/01/2017 Aceptado: 28/08/2017 